\title{
Berichtigung zu der Arbeit
}

\section{„Gleichungen in freien Produkten mit Amalgam“6}

\author{
Math. Z. 173, 1-12 (1980) \\ Gerhard Rosenberger \\ Abteilung Mathematik der Universität, Postfach 500500 , \\ D-4600 Dortmund 50, Bundesrepublik Deutschland
}

Die Formulierung von Satz (2.11) enthält einen Fehler. Dies wurde auch von J. Howie in einem Brief vom 20. Mai 1981 bemerkt.

Richtig muB Satz (2.11) folgendermaßen lauten:

,Sei $G=* H_{i \in I}$ freies Produkt der Gruppen $H_{i}$, $i \in I$. Sel $F$ freie Gruppe vom Rang $n \geqq 1$ mit der geordneten Basis $\left\{x_{1}, \ldots, x_{n}\right\}$ und $w_{0}=w\left(x_{1}, \ldots, x_{n}\right)$ ein reguläres Wort in F. Sei $\varphi$ ein Homomorphismus von $F$ in $G$ derart, daß $U .=\varphi(X)$ regulär und $\varphi(w)=1$ ist. Dann ist das Paar $(w, U)$ frei äquivalent (induziert von einem Automorphismus $\alpha$ von $F$ ) zu einem Paar $\left(w^{\prime}, U^{\prime}\right)=\left(\alpha(w), \alpha^{-1}(U)\right)$ mit den folgenden Eigenschaften:

(2.12) $w^{\prime}=r_{1} w_{1} r_{1}^{-1} \ldots r_{k} w_{k} r_{k}^{-1}$ für ein $k \geqq 0$, wobei $r_{i}, w_{i}(1 \leqq i \leqq k)$ frei reduzierte Worte in $x_{1}, \ldots, x_{n}$ sind.

(2.13) Für jedes $i(1 \leqq i \leqq k)$ ist $\varphi \alpha^{-1}\left(w_{i}\right)=1$.

(2.14) Für jedes $i(1 \leqq i \leqq k)$ gibt es ein $y_{i} \in I$ und ein $g_{i} \in G$ derart, daß $\varphi \alpha^{-1}\left(x_{j}\right) \in g_{i} H_{v_{i}} g_{i}^{-1}$ ist für jedes $x_{j}$, welches in $w_{i}$ vorkommt.

Ist darüberhinaus $\varphi$ ein Epimorphismus, so können wir $g_{i}=1$ für alle $i(1 \leqq i \leqq k)$ annehmen."

Der Unterschied liegt in Eigenschaft (2.12); die ursprüngliche Version von Satz (2.11) ist richtig, falls $w$ quadratisches Wort in $F$ ist (vgl. die Arbeiten [3] und [12] von Lyndon bzw. Zieschang). Für ein beliebiges $w$ kann aber zum Beispiel schon folgende Situation eintreten: $w^{\prime}=w_{1} w_{2}$ mit $w_{1}=w_{1}\left(x_{1}, x_{2}\right)$ und $w_{2}=x_{3} w_{1} x_{3}^{-1}$. Derartige Möglichkeiten wurden im Beweis von Satz (2.11) ausfuihrlich beriicksichtigt. Bei der Formulierung wurde allerdings leider gedankenlos die Version für ein quadratisches Wort $w$ übernommen (vgl. [3]). Dadurch ändert sich in den Beweisen überhaupt nichts. Für die in der Arbeit angegebenen vier Folgerungen sowie für den Rest der Arbeit ist der Fehler in der Formulierung von Satz (2.11) belanglos. 